B. KAŹMIERCZAK (Warszawa)

\title{
SOME EXISTENCE THEOREMS FOR NONLOCAL ELLIPTIC SYSTEMS. APPLICATION TO LASER PLASMA
}

Abstract. We formulate some existence theorems for systems of elliptic equations with nonlocal terms. The proofs are based on the invariant region method. The results are applied to a multitemperature model of laser sustained plasma.

1. Introduction. A variety of phenomena in physics, nuclear reactor theory, biology, medicine, chemistry etc. may be described by nonlinear reaction-diffusion systems. One of the most constructive methods of proving existence of their solutions is based on invariant rectangle (or generally invariant region) theorems ([1], [4], [16], [19]-[21]). Another method utilizes the notion of sub- and supersolution pairs ([3], [6], [10]). In this paper we consider elliptic equations with nonlocal nonlinear source terms.

In our case the necessity to take nonlocal terms into consideration has originated from a model describing energy phenomena in laser sustained plasma. The basic aim of this paper is to apply the abstract theorems formulated in Section 3 to such a model. In Section 4 we will analyze the existence of solutions (and some of their properties) for the following system of equations:

$$
\begin{aligned}
& L_{1}\left(x, u_{1}\right) u_{1}-v_{1}\left(x, u_{1}, \nabla u_{1}\right) \cdot \nabla u_{1} \\
& \quad+\gamma\left(u_{1}\right) I \Psi\left(u_{1}\right)(x)-G\left(u_{1}\right)-\sum_{j \neq 1}^{m} \sigma_{1 j}(u)\left(u_{1}-u_{j}\right)=0,
\end{aligned}
$$

2000 Mathematics Subject Classification: Primary 35J55, 35Q72.

Key words and phrases: elliptic equations, reaction-diffusion systems, nonlocal terms, a priori estimates, sub- and supersolution method.

This paper was partially supported by KBN grant No 7T07A00919. 


$$
L_{i}\left(x, u_{i}\right) u_{i}-v_{i}\left(x, u_{i}, \nabla u_{i}\right) \cdot \nabla u_{i}-\sum_{j \neq i} \sigma_{i j}(u)\left(u_{i}-u_{j}\right)=0,
$$

for $x \in \Omega \subset \mathbb{R}^{3}, i=2, \ldots, m, u=\left(u_{1}, \ldots, u_{m}\right)$, with boundary conditions

$$
u_{j}(x)=0, \quad j=1, \ldots, m, \quad x \in \partial \Omega .
$$

Here $u_{i}, i=2, \ldots, m$, is the temperature of the $i$ th component of the ionized gas, whereas $u_{1}$ denotes the temperature of the light (electron) component. $I$ denotes the asymptotic intensity of laser radiation. $L_{j}\left(x, u_{j}\right), j=1, \ldots, m$, are uniformly elliptic operators. The term $\Psi\left(u_{1}\right)(x)$ is nonlocal and models the absorption of energy of the laser beam during its passage through the ionized gas. A more detailed form of $\Psi\left(u_{1}\right)(x)$ will be given in Section 4 .

2. Invariant region theorems. The notion of invariant region for a system of parabolic equations with local terms has been widely exploited by many authors (see e.g. [1], [3], [6], [9], [16], [18], [19], [21]). Loosely speaking this is a region in the space of dependent variables having the following property: if the initial and boundary values lie in this region then the values of a solution, if it exists, also lie in it. By using standard methods one can prove that the existence of such a region implies the existence of a solution. A similar notion can be used in the case of systems of elliptic equations. In this case we assume that the boundary values of the Dirichlet problem lie in such a region ([6], [9], [18], [19]). A sine qua non condition for applying these methods is to have appropriate a priori estimates. In this work we put aside the question of possibility of such estimates. This property will be just assumed below. In the case of laser sustained plasma (Section 4) they are almost obvious. Such methods are very constructive. Besides the existence result they provide us with some information about the solution, because it is "contained" in the chosen region.

Below we will concentrate on the following system of elliptic equations containing, in general, nonlocal terms:

$$
L_{i}(x, u(x), \partial u(x)) u_{i}(x)=\mathcal{F}_{i}(u)(x), \quad x \in \Omega,
$$

with boundary conditions

$$
u_{i}(x)=\phi_{i}(x), \quad x \in \partial \Omega,
$$

where $i=1, \ldots, m \geq 1$ and $u:=\left(u_{1}, \ldots, u_{m}\right)$. For notational convenience we have set $\nabla u \equiv \partial u$. We make the following assumptions:

Assumption 1. $\Omega$ is a bounded open region in $\mathbb{R}^{n}, n \geq 1$, with $C^{2+\alpha}$ boundary. For $i \in\{1, \ldots, m\}$ the function $\phi_{i}$ is in $C^{2+\alpha}(\bar{\Omega})$, where $\bar{\Omega}$ denotes the closure of $\Omega$ and $\alpha \in(0,1)$.

Assumption 2. Each $L_{i}$ is of the form

$$
L_{i}(x, u, \partial u)=a_{k j}^{i}(x, u, \partial u) \partial_{k} \partial_{j},
$$


where $a_{k j}^{i}(x, u, \partial u)$ is symmetric with respect to $k, j$. For all $i \in\{1, \ldots, m\}$, the operators $L_{i}$ are uniformly elliptic in $\bar{\Omega}$, i.e. there exist continuous functions $\nu(x, u, p)>0$ and $\mu(x, u, p)>0$ such that for all $\xi \in \mathbb{R}^{n}, \xi \neq 0$,

$$
\nu(x, u, p) \xi^{2} \leq \sum_{j, k} a_{j k}^{i}(x, u, p) \xi_{j} \xi_{k} \leq \mu(x, u, p) \xi^{2},
$$

and $\mu(x, u, p) \nu(x, u, p)^{-1} \leq \mu_{1}<\infty$ for all $x \in \bar{\Omega}$, all $u \in \mathbb{R}^{m}$ and all $p \in \mathbb{R}^{m n}$. The coefficients $a_{k j}^{i}(x, u, \partial u)$ are of class $C^{\alpha}($ with $\alpha \in(0,1))$ on every bounded subset of $\bar{\Omega} \times \mathbb{R}^{m} \times \mathbb{R}^{m n}$.

Assumption 3. $\mathcal{F}_{i}, i \in\{1, \ldots, m\}$, is a mapping from $C^{2}(\bar{\Omega}) \times \ldots \times$ $C^{2}(\bar{\Omega})$ ( $m$ times) to $C^{\alpha}(\bar{\Omega}), \alpha \in(0,1)$.

Let $\Sigma:=\prod_{i=1}^{m}\left(a_{i}, b_{i}\right)$ with $a_{i}, b_{i} \in \mathbb{R}, a_{i}<b_{i}$.

Assumption 4. $\phi:=\left(\phi_{1}, \ldots, \phi_{m}\right): \bar{\Omega} \rightarrow \mathbb{R}^{m}$ and $\phi(x) \in \bar{\Sigma}$ for every $x \in \partial \Omega$.

Assumption 5. For every bounded open subset $W \subset \mathbb{R}^{m}$ there exists a positive number $\eta(\Omega, \phi, W)$ such that every $C^{2}\left(\bar{\Omega}, \mathbb{R}^{m}\right)$ solution $u$ of problem (4)-(5) with values in the closure of $W$ satisfies

$$
\|u\|_{2}:=\|\partial u\|_{C^{0}(\bar{\Omega})}+\left\|\partial^{2} u\right\|_{C^{0}(\bar{\Omega})} \leq \eta,
$$

where $\partial^{2} u$ is the matrix of second derivatives of $u$.

Below, for simplicity, we will often use the symbol $C^{\alpha}$ to denote the function space $C^{\alpha}(\bar{\Omega}) \times \ldots \times C^{\alpha}(\bar{\Omega})$ (m times).

Remark 1. Assumption 5 is satisfied when all $L_{i}$ are the same and do not depend on $\partial u$, whereas $\mathcal{F}_{i}$ can be represented by local functions $f_{i}=f_{i}(x, u, p)$, which are of class $C^{1}$ and

$$
\left|f_{i}(x, u, p)\right|<c(u)\left(\|p\| \cdot\left\|p_{i}\right\|+o\left(\|p\|^{2}\right)\right)
$$

for all $x \in \bar{\Omega}$ as $\|p\| \rightarrow \infty$ (see e.g. [13]). In the case of $L_{i}$ depending on $\partial u$, a priori estimates are much more difficult to achieve and some structure conditions are to be imposed. A great number of papers and books concern estimates for systems of equations in divergence form (see e.g. [12], [14]). However, in some situations, when the coefficients in the $i$ th equation depend only on $\partial u_{i}$, the theory for a single equation is sufficient (see [2], [13]).

Definition 1. Let $\mathcal{W}$ denote an open, bounded, convex subset of $\mathbb{R}^{m}$. Then by an outer normal vector at $u \in \partial \mathcal{W}$ we mean any unit vector $n(u)$ such that

$$
n(u) \cdot(U-u) \leq 0
$$

for all $U \in \overline{\mathcal{W}}$. 
A local existence result of invariant region type is contained in the following theorem.

THEOREM 1. Let Assumptions 1-2 and 4-5 be satisfied. Let $\mathcal{F}$ be represented by a (local) function $f(x, u, p):=\left(f_{1}(x, u, p), \ldots, f_{m}(x, u, p)\right)$ : $\bar{\Omega} \times \mathbb{R}^{m} \times \mathbb{R}^{m n} \rightarrow \mathbb{R}^{m}$ of class $C^{\alpha}$ on every compact subset of $\bar{\Omega} \times \mathbb{R}^{m} \times \mathbb{R}^{m n}$. Let $Q=\eta(\Omega, \phi, \Sigma)$. Suppose that for every $u \in \partial \Sigma$ there exists an outer normal vector $n(u)=\left(n_{1}(u), \ldots, n_{m}(u)\right)$ such that

$$
n(u) \cdot f(x, u, p) \geq 0
$$

for all $x \in \bar{\Omega}$ and all $p \in \mathbb{R}^{m n}$ such that $\|p\| \leq Q$ and $\sum_{i=1}^{m} p_{i j} n_{i}(u)=0$. Then problem (4)-(5) has a solution $u: \bar{\Omega} \rightarrow \bar{\Sigma}$ and $u \in C^{2+\alpha}(\bar{\Omega})$.

This result can be generalized to the case of equations with nonlocal terms.

TheOREm 2. Let Assumptions 1-5 be satisfied. Let $Q=\eta(\Omega, \phi, \Sigma)$. Let the mapping $\mathcal{F}=\left(\mathcal{F}_{1}, \ldots, \mathcal{F}_{m}\right)$ be such that for every $u_{0} \in \partial \Sigma$ there exists an outer normal vector $n\left(u_{0}\right)=\left(n_{1}\left(u_{0}\right), \ldots, n_{m}\left(u_{0}\right)\right)$ such that

$$
n\left(u_{0}\right) \cdot \mathcal{F}(u)\left(x_{0}\right) \geq 0
$$

for all $x_{0} \in \bar{\Omega}$ and all $C^{2+\alpha}(\bar{\Omega})$ functions $u: \bar{\Omega} \rightarrow \bar{\Sigma}$ such that $u\left(x_{0}\right)=u_{0}$, $\|u\|_{2} \leq Q$ and $\sum_{i=1}^{m} \partial_{j} u_{i}\left(x_{0}\right) \cdot n_{i}\left(u_{0}\right)=0$. Then problem (4)-(5) has a solution $u: \bar{\Omega} \rightarrow \bar{\Sigma}$ and $u \in C^{2+\alpha}(\bar{\Omega})$.

REMARK 2. If $L_{i}=a_{k j}(x, u)$ independently of $i$, then the assumptions of Theorem 2 can be weakened. Namely, for $\Sigma$ we may take any open, bounded, convex set of $\mathbb{R}^{m}$ (see [19]).

Though the proof of Theorem 2 (and Theorem 1) may be carried out along the same lines as the proof of Theorem 8, p. 270 in [19], for the reader's convenience we give it in the Appendix.

3. Sub- and supersolutions theorems. In this section we will use Theorem 2 to prove some existence results for systems having, in general, nonlocal right hand side terms, using the method of barrier functions.

Assumption 6. For $i \in\{1, \ldots, m\}$, let

$$
\mathcal{F}_{i}(u)(x)=f_{i}(x, u(x), \partial u(x)) F_{i}(u)(x)+H_{i}(u)(x),
$$

where $f_{i}, F_{i}$ and $H_{i}$ are of class $C^{\alpha}$ and $f_{i}$ is a local function.

It occurs that it is sometimes much easier to find a pair of functions satisfying certain inequalities than to construct an invariant region in the phase space. 
Assumption 7. For $i \in\{1, \ldots, m\}$ there exists a pair of functions $y_{i}$ and $Y_{i}$ of class $C^{2+\alpha}(\bar{\Omega})$ such that $y_{i}(x)<Y_{i}(x)$ for $x \in \bar{\Omega}$ and $y_{i}(x)$ $\leq \phi_{i}(x) \leq Y_{i}(x)$ for $x \in \partial \Omega$.

Let $W_{y}=\prod_{i=1}^{m}\left(c_{i}, d_{i}\right)$, with $c_{i}, d_{i} \in \mathbb{R}, c_{i}<d_{i}$, be such that the values of $y_{i}$ and $Y_{i}$ lie in $\left(c_{i}, d_{i}\right)$.

Assumption 8. Let $\beta=\eta\left(\Omega, \phi, W_{y}\right)$. We suppose that, for all $i \in$ $\{1, \ldots, m\}$,

$$
\begin{gathered}
L_{i}\left(x, u_{1}(x), \ldots, y_{i}(x), \ldots, u_{m}(x), \partial u_{1}(x), \ldots, \partial y_{i}(x), \ldots, \partial u_{m}(x)\right) y_{i}(x) \\
\geq f_{i}\left(x, u_{1}(x), \ldots, y_{i}(x), \ldots, u_{m}(x), \partial u_{1}(x), \ldots, \partial y_{i}(x), \ldots, \partial u_{m}(x)\right) \\
\times F_{i}\left(u_{1}, \ldots, u_{i}, \ldots, u_{m}\right)(x)+H_{i}\left(u_{1}, \ldots, u_{i}, \ldots, u_{m}\right)(x), \\
L_{i}\left(x, u_{1}(x), \ldots, Y_{i}(x), \ldots, u_{m}(x), \partial u_{1}(x), \ldots, \partial Y_{i}(x), \ldots, \partial u_{m}(x)\right) Y_{i}(x) \\
\leq f_{i}\left(x, u_{1}(x), \ldots, Y_{i}(x), \ldots, u_{m}(x), \partial u_{1}(x), \ldots, \partial Y_{i}(x), \ldots, \partial u_{m}(x)\right)(x) \\
\times F_{i}\left(u_{1}, \ldots, u_{i}, \ldots, u_{m}\right)(x)+H_{i}\left(u_{1}, \ldots, u_{i}, \ldots, u_{m}\right)(x)
\end{gathered}
$$

for all $x \in \bar{\Omega}$ and all $u_{\nu}, \nu \in\{1, \ldots, m\}, \nu \neq i$, satisfying the inequalities

$$
y_{\nu}(x) \leq u_{\nu}(x) \leq Y_{\nu}(x)
$$

and such that

$$
\|u\|_{2} \leq \beta .
$$

TheOREm 3. Suppose that Assumptions 1-8 are satisfied. Then there exists a $C^{2+\alpha}(\bar{\Omega})$ solution $\left(u_{1}(x), \ldots, u_{m}(x)\right)$ of problem $(4)-(5)$ such that $y_{i}(x) \leq u_{i}(x) \leq Y_{i}(x)$ for $x \in \bar{\Omega}$.

Proof. Let us change the variables $u_{i} \rightarrow u_{i}^{*}$, where

$$
u_{i}=u_{i}^{*} Y_{i}+\left(1-u_{i}^{*}\right) y_{i} \text {. }
$$

Then

$L_{i}(x, u, \partial u) u_{i}=L_{i}^{*}(x, u, \partial u) u_{i}^{*}+\left(1-u_{i}^{*}\right) L_{i}(x, u, \partial u) y_{i}+u_{i}^{*} L_{i}(x, u, \partial u) Y_{i}$, where

$$
L_{i}^{*}(x, u, \partial u)=\left(Y_{i}-y_{i}\right) L_{i}(x, u, \partial u)+2 a_{j k}^{i}(x, u, \partial u)\left\{\partial_{k}\left(Y_{i}-y_{i}\right)\right\} \partial_{j} .
$$

Thus (4) is equivalent to the equation

$$
L_{i}\left(x, u\left(u^{*}(x)\right), \partial u\left(u^{*}(x)\right)\right) u_{i}^{*}(x)=\left(Y_{i}(x)-y_{i}(x)\right)^{-1} \mathcal{F}_{i}^{*}\left(u^{*}\right)(x)
$$

and the boundary conditions (5) are equivalent to

$$
u_{i}^{*}(x)=\left[\phi_{i}(x)-y_{i}(x)\right]\left[Y_{i}(x)-y_{i}(x)\right]^{-1}=: \varrho_{i}(x),
$$

for $i=1, \ldots, m$,

$$
\mathcal{F}_{i}^{*}\left(u^{*}\right)(x)=\mathcal{F}_{i}\left(u\left(u^{*}\right)\right)(x)+\mathcal{R}\left(u^{*}\right)(x),
$$


where

$$
\begin{aligned}
\mathcal{R}\left(u^{*}\right)(x)= & -2 a_{j k}^{i}\left(x, u\left(u^{*}(x)\right), \partial u\left(u^{*}(x)\right)\right)\left\{\partial_{k}\left(Y_{i}(x)-y_{i}(x)\right)\right\} \partial_{j} u_{i}^{*}(x) \\
& -\left(1-u_{i}^{*}(x)\right) L_{i}\left(x, u\left(u^{*}(x)\right), \partial u\left(u^{*}(x)\right)\right) y_{i}(x) \\
& -u_{i}^{*}(x) L_{i}\left(x, u\left(u^{*}(x)\right), \partial u\left(u^{*}(x)\right)\right) Y_{i}(x),
\end{aligned}
$$

and according to $(9)$,

$$
u_{j}\left(u_{j}^{*}\right)=u_{j}^{*} Y_{j}+\left(1-u_{j}^{*}\right) y_{j} .
$$

To apply Theorem 2 we will set $\Sigma=\Lambda$, where $\Lambda$ is an open rectangle in the $u^{*}$-space whose vertices have coordinates equal to 0 or 1 .

Now, we can determine a unit (though nonunique) vector field $n\left(u^{*}\right)$ on $\partial \Lambda$ satisfying the conditions of Definition 1 and thus being a field of vectors normal to $\partial \Lambda$ at every point of it. Namely, if $u^{*}$ belongs to an open side $I_{+1 i}:=\left(t_{1}, \ldots, t_{i-1}, 1, t_{i+1}, \ldots, t_{m}\right)$ or $I_{-1 i}\left(t_{1}, \ldots, t_{i-1}, 0, t_{i+1}, \ldots, t_{m}\right)$ with $t_{j} \in(0,1)$, then let $n\left(u^{*}\right)=n_{i}$ or $n\left(u^{*}\right)=-n_{i}$, where $n_{i}$ denotes the unit vector directed along the $i$ th axis. For all the other $u^{*}$ there exists the smallest $i \in\{1, \ldots, m\}$ such that $u^{*}$ belongs to the intersection of the closures of the sides $I_{\chi i}$ and $I_{\tilde{\chi} j}, i<j, \chi, \widetilde{\chi} \in\{-1,+1\}$. Then let $n\left(u^{*}\right):=$ $\chi n_{i}$. We must check that for $\Sigma=\Lambda$, for this outer normal vector field and for system (10)-(11), Assumptions 1-5 are satisfied with respect to $u_{i}^{*}$ and $\varrho$ (in place of $u$ and $\phi)$. First, note that, due to the form of $(9)$ and Assumption 5, we have a priori estimates for any function $u^{*}$ satisfying the system (10)-(11), namely $\left\|u^{*}\right\|_{2} \leq \eta(\Omega, \varrho, \Lambda)=Q^{*}$.

Now we check that condition (7) is satisfied. So, suppose that $u_{0}^{*}=$ $u^{*}\left(x_{0}\right) \in \partial \Lambda, x_{0} \in \Omega$. Then $n\left(u_{0}^{*}\right)= \pm n_{i}$, where $n_{i}$ is the unit vector directed along the $i$ th axis. Suppose that $n\left(u_{0}^{*}\right)=n_{i}$ for some $i \in\{1, \ldots, m\}$. Then $u_{0 i}^{*}=1$ and the value of $n\left(u^{*}\left(x_{0}\right)\right) \cdot \mathcal{F}^{*}(u)\left(x_{0}\right)$ for $\partial_{j} u_{i}^{*}\left(x_{0}\right)=0$ is equal to

$$
\begin{aligned}
\mathcal{F}_{i}^{*}\left(u^{*}\right)\left(x_{0}\right)= & f_{i}\left(x_{0}, U_{i}\left(u^{*}\right)\left(x_{0}\right), \partial U_{i}\left(u^{*}\right)\left(x_{0}\right)\right) F\left(u^{*}\right)\left(x_{0}\right) \\
& +H\left(u^{*}\right)\left(x_{0}\right)-L_{i}\left(x_{0}, U_{i}\left(u^{*}\right)\left(x_{0}\right), \partial U_{i}\left(u^{*}\right)\left(x_{0}\right)\right) Y_{i}\left(x_{0}\right),
\end{aligned}
$$

where $U_{i}\left(u^{*}\right)=\left(u_{1}\left(u_{1}^{*}\right), \ldots, Y_{i}, \ldots, u_{m}\left(u_{m}^{*}\right)\right)$ with $u_{j}\left(u_{j}^{*}\right)$ determined by $(9)$ and $u_{j}^{*}$ such that $0 \leq u_{j}^{*}(x) \leq 1, j \neq i$, and $\left\|u^{*}\right\|_{2} \leq \eta(\Omega, \varrho, \Lambda)$. According to Assumption 7 this value is nonnegative for all $x_{0} \in \bar{\Omega}$. Thus condition (7) is satisfied. In the same way we can prove that this condition remains valid for $n\left(u^{*}\right)=-n_{i}$. The theorem is proved.

REMARK 3. If the inequalities satisfied by $L_{i}$ in Assumption 8 are strict, then in Assumption 7 we can demand only $y_{i}(x) \leq Y_{i}(x)$. This follows from the fact that in this case we can replace $y_{i}$ and $Y_{i}$ by functions $\widehat{y}_{i}$ and $\widehat{Y}_{i}$ (for example by adding constants of sufficiently small absolute value) such that $\widehat{y}_{i}<\widehat{Y}_{i}$ and Assumption 7 is satisfied (for $\widehat{y}_{i}$ and $\widehat{Y}_{i}$ ). 
In Section 4 we will consider equations of laser sustained plasma, in which the mappings $H_{i}$ are local functions. Assumption 8 may then be weakened.

Assumption 9. For all $i \in\{1, \ldots, m\}$, let

$$
L_{i}(x, u, \partial u)=a_{k j}^{i}\left(x, u_{i}, \partial u_{i}\right) \partial_{k} \partial_{j}
$$

and

$$
\mathcal{F}_{i}(u)(x)=f_{i}(x, u(x), \partial u(x)) F_{i}(u)(x)+H_{i}(x, u(x), \partial u(x)),
$$

i.e. let $f_{i}$ and $H_{i}$ be local functions. Let $\beta=\eta\left(\Omega, \phi, W_{y}\right)$ and suppose that, for all $i \in\{1, \ldots, m\}$,

$$
\begin{aligned}
L_{i}\left(x, y_{i}(x), \partial y_{i}(x)\right) & \\
\geq & f_{i}\left(x, u_{1}(x), \ldots, y_{i}(x), \ldots, u_{m}(x), \partial u_{1}(x), \ldots, \partial y_{i}(x), \ldots, \partial u_{m}(x)\right) \\
& \times F_{i}\left(u_{1}, \ldots, u_{i}, \ldots, u_{m}\right)(x) \\
& +H_{i}\left(x, u_{1}(x), \ldots, y_{i}(x), \ldots, u_{m}(x), \partial u_{1}(x), \ldots, \partial y_{i}(x), \ldots, \partial u_{m}(x)\right)
\end{aligned}
$$$$
L_{i}\left(x, Y_{i}(x), \partial Y_{i}(x)\right)
$$$$
\leq f_{i}\left(x, u_{1}(x), \ldots, Y_{i}(x), \ldots, u_{m}(x), \partial u_{1}(x), \ldots, \partial Y_{i}(x), \ldots, \partial u_{m}(x)\right)
$$$$
\times F_{i}\left(u_{1}, \ldots, u_{i}, \ldots, u_{m}\right)(x)
$$$$
+H_{i}\left(x, u_{1}(x), \ldots, Y_{i}(x), \ldots, u_{m}(x), \partial u_{1}(x), \ldots, \partial Y_{i}(x), \ldots, \partial u_{m}(x)\right),
$$

for all $x \in \bar{\Omega}$ and all $u_{\nu}, \nu \in\{1, \ldots, m\}, \nu \neq i$, satisfying the inequalities

$$
y_{\nu}(x) \leq u_{\nu}(x) \leq Y_{\nu}(x)
$$

and such that

$$
\|u\|_{2} \leq \beta .
$$

TheOREm 4. Let Assumptions 1-7 and Assumption 9 hold. Then there exists a $C^{2+\alpha}(\bar{\Omega})$ solution $\left(u_{1}(x), \ldots, u_{m}(x)\right)$ to problem $(4)-(5)$ such that $y_{i}(x) \leq u_{i}(x) \leq Y_{i}(x)$ for all $x \in \bar{\Omega}$.

This theorem may be proved similarly to Theorem 3 .

4. Application to equations of laser plasma. Let us examine the Dirichlet problem connected with stationary laser sustained plasma. The model considered below was initiated in the paper of Eckhaus, van Harten and Peradzyński [5] and developed, in a sense, in [8]. However, in contrast to [8] we will work in the spaces of Hölder continuous functions. The schematic experimental LSP (laser sustained plasma) setting is shown in Fig. 1 of [8]. The incoming cold gas is entering the light cone of laser radiation. Here it gains energy in the process of heat conduction and directly from the laser radiation in the process of Inverse Brehmsstrahlung. The crucial element of the model is introducing the effect of laser light absorption by the ionized 
gas. So, let $W_{0}$ denote the ideal light cone, i.e. the light cone generated by an ideal lens. It is determined by a focal point $F \in \Omega$, which is a focus of the lens, the axis of the lens, which may be identified with the $z$-axis, and its angle $\theta$. Thus in cylindrical coordinates $(z, \varrho, \varphi)$ centered at $F$,

$$
W_{0}=\left\{(z, \varrho, \varphi): \cos ^{2}(\theta) z^{2} \geq \varrho^{2} \sin ^{2}(\theta)\right\} .
$$

(Cf. Fig. 1 in [8].) Because we want to work in spaces of smooth functions we will diffuse the boundary of $W_{0}$. This will also take into account the fact that the real focus of the lens always has a nonzero diameter. First, define an auxiliary cut-off type function. Namely, for $k>0$, let $\widetilde{\psi}(k, r) \equiv 0$ for $r \leq 0, \widetilde{\psi}(k, r) \equiv 1$ for $r \geq 1$ and

$$
\widetilde{\psi}(k, r)=\frac{\eta_{k}(r)}{\eta_{k}(r)+\eta_{k}(1-r)} \quad \text { for } r \in(0,1),
$$

where $\eta_{k}(r)=\exp (-k / r)$ for $r \in(0, \infty)$, and $\eta_{k}(r) \equiv 0$ for $r \in(-\infty, 0]$. For $b>c$, let

$$
\psi(k, c, b, x)=\widetilde{\psi}\left(k, \frac{x-c}{b-c}\right) .
$$

The function $\psi(k, c, b, x)$ is of class $C^{\infty}$. As

$$
\widetilde{\psi}\left(k, \frac{x-c}{b-c}\right)+\widetilde{\psi}\left(k, \frac{b-x}{b-c}\right)=1,
$$

we have

$$
\int_{b}^{c} \widetilde{\psi}\left(k, \frac{x-c}{b-c}\right) d x=\frac{b-c}{2} .
$$

Now, let $a$ be of the order of the physical focus of the lens. Consider a cylinder $K=\left\{(z, \varrho, \varphi): \varrho \leq \frac{3}{2} a t, z \in[-a, a]\right\}$, where $t=\tan (\theta)$. Let $\psi_{a}(z)=\psi(1, a, 2 a, z)$. Let $W:=K \cup W_{0} \cup L_{1} \cup L_{2}$, where

$$
\begin{aligned}
L_{1} & =\left\{(z, \varrho, \varphi): z \in[a, 2 a], \varrho \leq t \int_{2 a}^{z} \psi_{a}(s) d s+2 t a\right\} \\
L_{2} & =\left\{(z, \varrho, \varphi): z \in[-2 a,-a], \varrho \leq-t \int_{-2 a}^{z} \psi_{a}(-s) d s+2 t a\right\} .
\end{aligned}
$$

To every point in the light cone we may assign a unique line. There is a one-to-one correspondence between these lines and the points $\left(0, \varrho_{0}, \varphi_{0}\right)$ of the circle $\{(z, \varrho, \varphi): z=2 a, \varrho \leq 2 a t\}$. Every line can be parametrized by $z$ through the relations 


$$
\begin{array}{ll}
\varrho(z)=\frac{\varrho_{0}}{2 a} z, \quad \varphi=\varphi_{0} & \text { for } z^{2}>4 a^{2}, \\
\varrho(z)=\frac{\varrho_{0}}{2 a} \int_{2 a}^{z} \psi_{a}(s) d s+\varrho_{0}, \quad \varphi=\varphi_{0} & \text { for } a \leq z \leq 2 a, \\
\varrho(z)=-\frac{\varrho_{0}}{2 a} \int_{-2 a}^{z} \psi_{a}(-s) d s+\varrho_{0}, \quad \varphi=\varphi_{0} & \text { for }-2 a \leq z \leq-a, \\
\varrho(z) \equiv \varrho_{0}, \quad \varphi=\varphi_{0} & \text { for } z^{2}<a^{2} .
\end{array}
$$

These lines are smooth and they differ from straight lines only in the small set $K \cup L_{1} \cup L_{2}$. In $K$ they become segments parallel to the $z$-axis. Now, for a given $u \in C^{2+\alpha}$, define the absorption term:

$$
\begin{array}{ll}
\Psi_{0}(u)(x)=\mathcal{K}(x) \exp \left(-\int_{l_{x}(x) \cap \Omega} \gamma(u(l)) d l\right), & x \in W, \\
\Psi_{0}(u)(x) \equiv 0, & x \notin W,
\end{array}
$$

where we integrate along the light line corresponding to the point $x$ (up till the point $x$ ) in the direction of increasing $z$. (We assume that the laser light comes from $z=-\infty$.) In (14), $\gamma$ is the absorption coefficient of the laser light. The factor $\mathcal{K}(x)$ models the effect of focusing the radiation beam by the lens. We tacitly assume that the light rays in plasma do not change when compared with those in vacuum.

As the boundary of $W$ is smooth, we can make $\Psi$ a Hölder continuous function of $x \in \bar{\Omega}$ by taking

$$
\Psi(u)(x)=\left\{1-\psi_{W}(x)\right\} \Psi_{0}(u)(x),
$$

where $\psi_{W}(x)=\psi\left(1,0, \varepsilon_{W}, d_{W}(x)\right), \varepsilon_{W}>0$ is some constant, and $d_{W}(x)$ denotes the distance of $x$ to $Y(x)$, the point of $\partial W$ nearest to $x$. If $\varepsilon_{W}$ is taken sufficiently small, then this function is well defined. (We take $d_{W}(x) \leq 0$ for $x \in W$, so $\psi_{W}(x)=0$ for $x \in W$.) We will also take into account the process of energy losses through radiation. The phenomenological function $G(u)$ which describes this process is an increasing function of $u$, it is rather small for low temperatures and rises abruptly for large $u$.

Thus, we describe the mean temperature field $u$ in laser sustained plasma by the equation

$$
\Delta u-\vec{v}(x) \cdot \nabla u+\gamma(u) I \Psi(u)(x)-G(u)=0, \quad x \in \Omega,
$$

with the zero boundary conditions

$$
u(x)=0, \quad x \in \partial \Omega .
$$

(We have chosen the constant coefficient elliptic operator only for the sake of clarity. More general elliptic operators will be considered below.) 
Assumption 10. $\vec{v}: \bar{\Omega} \rightarrow \mathbb{R}^{3}$ is of class $C^{1}$. The functions $\gamma, G: \mathbb{R} \rightarrow \mathbb{R}$ and $\mathcal{K}: \bar{\Omega} \rightarrow \mathbb{R}$ are of class $C^{1} \cdot \gamma(u) \equiv 0$ for $u \in(-\infty, 0], \gamma^{\prime}(u)>0$ for $u \in\left(0, u_{*}\right), u_{*}>0, \gamma(u)<\gamma^{*}<\infty$ for all $u \in(0, \infty), \gamma(u) \geq \gamma>0$ for $u \in\left[u_{*}, \infty\right) . G(u) \equiv 0$ for $u \in(-\infty, 0], 0<G(u)$ for all $u \in(\overline{0}, \infty)$, $G(u) \rightarrow \infty$ as $u \rightarrow \infty$. $\Omega \subset \mathbb{R}^{3}$ satisfies Assumption 1 .

It follows from Assumption 10 that for any fixed intensity $I$ of the laser radiation and every $x$ inside the light cone $W$, there exists a unique $u(I, x)$ satisfying the equation $\gamma(u) \Psi(u)(x) I-G(u)=0$ and such that $\gamma(u) \Psi(u)(x)-G(u)<0$ for all $u>u(I, x)$.

Existence of a priori estimates is obvious. Namely, if $f$ is bounded in $\Omega$, then any solution to the equation $\Delta u(x)-\vec{v}(x) \cdot \nabla u=f(x)$ satisfying the zero boundary conditions belongs to $W_{p}^{2}(\Omega)$ for all $p \in(2, \infty)$, hence (due to the imbedding theorem) also belongs to the space $C^{1+\tau}$ for all $\tau \in(0,1)$. Thus, by a "bootstrap" argument we infer that Assumption 5 is satisfied.

To prove the existence of solutions to problem (16)-(17) we will construct appropriate sub- and supersolutions. Let $B$ be a given $C^{3}$ domain such that $\bar{B} \subseteq(W \backslash \partial W) \cap \Omega$. We know that for all $x \in \Gamma_{\mu}=\{x \in \bar{B}: \operatorname{dist}(x, \partial B) \leq \mu\}$, where $\mu>0$ depends on $B$, the function $\operatorname{dist}(x, \partial B)$ is of clas $C^{3}$ (see [7], Lemma 14.16). (This time, unlike the case of the function $\Psi$ defining the absorption term, we have taken $\operatorname{dist}(x, \partial B)$ to be positive for $x \in B$.) Let $h \in(0, \mu / 2]$ and let

$$
\psi_{B}(h, x)= \begin{cases}0 & \text { for } x \in B, \\ \psi(h, d(x)) & \text { for } x \in \Gamma_{h}, \\ 1 & \text { for } x \in B \backslash \Gamma_{h},\end{cases}
$$

where $\psi(h, d(x))=\widetilde{\psi}\left(1, d(x) h^{-1}\right), d(x)=\operatorname{dist}(x, \partial B)$. Our candidate for a subsolution will be

$$
y(J, x)=\psi_{B}(h, x) J,
$$

where $J$ is a constant. We have

$$
\Delta y(J, x)-\vec{v}(x) \cdot \nabla y(J, x)=J \Delta \psi_{B}-J \vec{v}(x) \cdot \nabla \psi_{B} .
$$

Note that if $\vec{n}(x)$ is an outer normal to $\partial B$ at $x$ then $\nabla \psi_{B}(h, x) \cdot \vec{n}(x) \leq 0$. Moreover, due to the form of $\widetilde{\psi}$ we have $\psi^{\prime}(h, d(x))=O\left(d^{2}(x)\right) \psi^{\prime \prime}(h, d(x))$ as $d(x) \searrow 0$, where $\psi^{\prime}$ denotes the derivative of $\psi$ with respect to $d=d(x)$. For $x \in \Gamma_{h}$ we have $\Delta \psi_{B}(h, x)=\psi^{\prime \prime}(h, d(x))+\psi^{\prime}(h, d(x)) \nabla \cdot \vec{n}(x)$, where $\vec{n}(x)$ is the unit vector normal to $\partial B$ at the unique point $y(x) \in \partial B$ such that $d(x)=|x-y(x)|$. As noted above this is a $C^{3}$ function on $\Gamma_{\mu}$. Thus $\Delta \psi_{B}>0$ for all $x \in \Gamma_{\mu_{*}}, x \notin \partial B$, for some $\mu_{*} \in(0, \mu)$ sufficiently small. Consequently, since $\psi_{B}(h, x)=O\left(d^{4}(x)\right) \psi^{\prime \prime}(h, d(x))$ as $d(x) \searrow 0$, we have $\Delta y(J, x)>0$ in $\Gamma_{\mu_{*}}, x \notin \partial B$. 
Our candidate for a supersolution will be

$$
Y(A, \varepsilon, x)=A K(x)+\varepsilon,
$$

where $K(x)$ is the positive (on $\Omega$ ) eigenfunction corresponding to the principal negative eigenvalue of $\Delta-\vec{v}(x) \cdot \nabla$ with respect to $\Omega$ with $\|K\|_{L^{2}(\Omega)}=1$ (see the theorems of Chapter VIII in [13]) and $\varepsilon>0$ can be taken arbitrarily small.

We are in a position to prove the following lemma.

Lemma 1. For arbitrary $J>0$ and $\varepsilon>0$ there exists $I^{*}>0$ such that for all $I>I^{*}$ and all $A>A^{*}(J, I, \varepsilon)$,

$$
\begin{gathered}
y(J, x)<Y(A, \varepsilon, x), \\
\Delta y(J, x)-\vec{v} \cdot \nabla y(J, x)+\gamma(y(J, x)) I \Psi(u)(x)-G(y(J, x)) \geq 0, \\
\Delta Y(A, \varepsilon, x)-\vec{v} \cdot \nabla Y(A, \varepsilon, x) \\
+\gamma(Y(A, \varepsilon, x)) I \Psi(u)(x)-G(Y(A, \varepsilon, x)) \leq 0,
\end{gathered}
$$

for all $x \in \bar{\Omega}$ and all $C^{2+\alpha}(\bar{\Omega})$ functions $u$ satisfying the inequality

$$
y(J, x) \leq u(x) \leq Y(A, \varepsilon, x) .
$$

Proof. Fix $J$. As already noted, there exists $\mu_{*}>0$ such that for all $x \in \Gamma_{\mu_{*}}, x \notin \partial B$, we have $\Delta y(J, r)>0$. Moreover, the dominating term in this quantity is $J \Delta \psi_{B}(h, x)=J \psi^{\prime \prime}(h, d(x))$ and the term $G(y(J, x))$ behaves like $G^{\prime}(y(J, x)) J \psi_{B}(h, x)=G^{\prime}(y(J, x)) J O\left(d^{4}(x)\right) \psi^{\prime \prime}(h, d(x))$, so by taking smaller $\mu_{*}$ if necessary we can achieve that $\Delta y(J, x)-\vec{v} \cdot \nabla y(J, x)+$ $\gamma(y(J, x)) I-G(y(J, x))>0$ for all $x \in \Gamma_{\mu_{*}}, x \notin \partial B$. Obviously, $\nabla y(J, x)$, $\Delta y(J, x)$ and $G(y(J, x))$ are bounded in $B$. According to Assumption 10 the function $\gamma(u)$ is bounded and, if $u_{2} \geq u_{1}$, then either $\gamma\left(u_{2}\right) \geq \gamma\left(u_{1}\right)$ or $\gamma\left(u_{2}\right) \geq \gamma$. So, for all $C^{2}(\bar{\Omega})$ functions $u$ such that $u(x) \geq y(J, x) \in \Omega$ we have $\gamma(y \overline{(J}, x)) \Psi(u)(x)>g_{J}>0$ in $B \backslash \Gamma_{\mu_{*}}$ and one can choose $I^{*}$ so large that for all $I>I^{*}$,

$$
\Delta y(J, x)-\vec{v} \cdot \nabla y(J, x)+\gamma(y(J, x)) I \Psi(u)(x)-G(y(J, x))>0
$$

in $\Omega$. Note that this holds independently of how fast $\gamma(u)$ tends to 0 as $u \searrow 0$. Now, for fixed $I>I^{*}$ we can find $A^{*}>0$ so large that for all $A>A^{*}$ we have $Y(A, \varepsilon, x)>y(J, x)$ for all $x \in \Omega$ and the function $Y(A, \varepsilon, x)$ satisfies inequality (23). This follows from the fact that (for finite $I$ ) the nonlocal term is globally bounded from above in view of Assumption 10. The proof of the lemma is complete.

By means of the last lemma and Theorem 4 we conclude that there exists a solution to problem (16)-(17) between the functions $y(J, x)$ and $Y(A, \varepsilon, x)$. So in order that the solution $u(x)$ obtained by the above method be larger than some given $U>0$ at some $x \in B$ we must take $I$ sufficiently large. Moreover, the following theorem holds. 
Theorem 5. For any $C^{3}$ domain $B$ with $\bar{B} \subset \Omega \cap(W \backslash \partial W)$, and any given number $U>0$ there exists $I_{B U}>0$ such that for all $I>I_{B U}$ there exists a solution $u \in C^{2+\alpha}$ of (16)-(17) such that $u(x)>U$ for all $x \in B$.

Proof. There exists a domain $B^{*}$ with closure lying in $(W \backslash \partial W) \cap \Omega$ such that $\bar{B} \subset B^{*}, B^{*}$ has a $C^{3}$ boundary and the distance between $B$ and $\partial B^{*}$ is larger than some positive number $\mu_{1}$. Let $y^{*}(J, x)=J \psi_{B^{*}}(h, x)$, where as before $\psi_{B^{*}}(h, x)=\psi(h, d(x)), d(x)=\operatorname{dist}\left(x, \partial B^{*}\right)$ and $J>U$. We may take $h$ so small that $\psi_{B^{*}}(h, x)$ is of class $C^{3}$ and $\psi_{B}(h, x) \equiv 1$ for all $x \in B$. Taking $I_{B U}$ sufficiently large we obtain the result corresponding to Lemma 1. This implies the existence of a suitable solution. The differences in the proof are purely technical.

Multitemperature model. In a multitemperature model, laser sustained plasma is described by a system of $m \geq 2$ equations of the form

$$
\begin{gathered}
L_{1}\left(x, u_{1}\right) u_{1}-\vec{v}_{1}\left(x, u_{1}, \nabla u_{1}\right) \cdot \nabla u_{1}+\gamma\left(u_{1}\right) I \Psi\left(u_{1}\right)(x) \\
-G\left(u_{1}\right)-\sum_{j \neq 1}^{m} \sigma_{1 j}(u)\left[u_{1}-u_{j}\right]=0, \\
L_{i}\left(x, u_{i}\right) u_{i}-\vec{v}_{i}\left(x, u_{i}, \nabla u_{i}\right) \cdot \nabla u_{i}-\sum_{j \neq i} \sigma_{i j}(u)\left[u_{i}-u_{j}\right]=0,
\end{gathered}
$$

for $x \in \Omega$, where $\Omega$ is a bounded open subset of $\mathbb{R}^{3}$ with $C^{2+\alpha}$ boundary, $i=2, \ldots, m, u=\left(u_{1}, \ldots, u_{m}\right)$, with boundary conditions

$$
u_{j}(x)=0, \quad x \in \partial \Omega, \quad j=1, \ldots, m
$$

(see e.g. [11] and the references therein). Here $u_{i}(i=2, \ldots, m)$ can be interpreted as the temperature of the $i$ th component of the ionized gas, whereas $u_{1}$ denotes the temperature of the light (electron) component. The terms $\sigma_{i j}(u)\left(u_{i}-u_{j}\right), i, j \in\{1, \ldots, m\}, j \neq i$, describe the energy transfer between the $i$ th and $j$ th component of the ionized gas. We assume that $L_{i}$ are uniformly elliptic operators in $\bar{\Omega}$, i.e. they satisfy Assumption 2. We use general elliptic operators instead of $\Delta$ to take into account the fact that diffusion coefficients may depend on position in space and on the components of $u$.

Assumption 11. For $i \in\{1, \ldots, m\}, \vec{v}_{i}: \bar{\Omega} \times \mathbb{R} \times \mathbb{R}^{3} \rightarrow \mathbb{R}^{3}$ is of class $C^{1}$. For $i \in\{1, \ldots, m\}, j \neq i, \sigma_{i j}: \mathbb{R}^{m} \rightarrow \mathbb{R}, \sigma_{i j} \in C^{1}, \sigma_{i j}(u)>0$ in the set $P_{u}=\left\{\left(u_{1}, \ldots, u_{m}\right): u_{1}>0, \ldots, u_{m}>0\right\}, \sigma_{i j}=\sigma_{i j}\left(u_{i}\right), i, j \neq 1$.

Assumption 12. For all $i \in\{1, \ldots, m\}$, all $x \in \bar{\Omega}$, all $u_{i} \in \mathbb{R}$ and all $p_{i} \in \mathbb{R}^{3}$,

$$
\left|\vec{v}_{i}\left(x, u_{i}, p_{i}\right) \cdot p_{i}\right| \leq m\left(u_{i}\right)\left|p_{i}\right|\left(1+\left|p_{i}\right|\right),
$$

where $m$ is a continuous function. 
A priori estimates for system (24)-(26) can be obtained by means of Lemma VIII.4.1 of [13] applied separately to each equation of the system. This can be done due to the fact that the nonlocal term in the first equation is globally bounded and we can treat it as a given function depending on $x$.

Let

$$
y(J, x)=J \psi_{B}(h, x),
$$

where $J$ is a constant and $\psi_{B}$ has the same meaning as before, i.e. $\psi_{B}(h, x)=$ $\psi(h, d(x))$.

If $\vec{n}(x)$ is an outer normal to $\partial B$ then by the definition of $\psi_{B}(h, x)$ we have $\vec{n}(x) \cdot \nabla \psi_{B}(h, x) \leq 0$. Let us also recall that

$$
\psi_{B}(h, x)=O\left(d^{2}(x)\right) \psi^{\prime}(h, d(x)), \quad \psi^{\prime}(h, d(x))=O\left(d^{2}(x)\right) \psi^{\prime \prime}(h, d(x))
$$

as $d(x) \searrow 0$, where $\psi^{\prime}$ denotes the derivative of $\psi$ with respect to $d=d(x)$. By the definition of $\psi$, it takes constant values on hypersurfaces which are equidistant from the boundary of $B$. Thus for $x$ sufficiently close to $\partial B$ the expression $\left[L_{1}(x, 0)-\vec{v}_{1}(x, 0,0) \cdot \nabla\right] J \psi_{B}(h, x)$ is equal to

$$
\begin{aligned}
J \sum_{i, j} a_{i j}^{1}(x, 0) n_{i}(y(x)) n_{j}(y(x)) \psi^{\prime \prime}(h, d(x))\left(1+O\left(d^{2}(x)\right)\right) & \\
& -J \sum_{i} v_{1 i}(x, 0,0) n_{i}(y(x)) \psi^{\prime}(h, d(x)),
\end{aligned}
$$

where $y(x)$ is the uniquely determined point $y(x) \in \partial B$ such that

$$
d(x)=|x-y(x)|,
$$

where as before $d(x)$ denotes the distance to $\partial \Omega$. This term is positive for $x \notin \partial B$, but sufficiently close to $\partial B$. Thus for some $0<h^{*}<h / 2$ sufficiently small and for $x$ such that $d(x)<h^{*}$ we have

$$
\begin{aligned}
& {\left[L_{1}\left(x, J \psi_{B}(h, x)\right)-\vec{v}_{1}\left(x, J \psi_{B}(h, x), J \nabla \psi_{B}(h, x)\right) \cdot \nabla\right] J \psi_{B}(h, x)} \\
& \quad+G\left(J \psi_{B}(h, x)\right)-\sum_{j \neq 1} \sigma_{1 j}^{*}(x) J \psi_{B}(h, x) \\
& \quad=\left[L_{1}(x, 0)-\vec{v}_{1}(x, 0,0) \cdot \nabla\right] J \psi_{B}(h, x)+O\left(J \psi_{B}(h, x)\right)+O\left(J\left|\nabla \psi_{B}(h, x)\right|\right) \\
& \geq \frac{1}{4} \psi^{\prime \prime}(h, d(x)) J .
\end{aligned}
$$

Here

$$
\sigma_{1 j}^{*}(x)=\max _{u_{k} \in\left[0, J \psi_{B}(h, x)\right], k=2, \ldots, m} \sigma_{1 j}\left(J \psi_{B}(h, x), u_{2}, \ldots, u_{m}\right) .
$$

Hence, as $\gamma(y)>0$ for all $y>0$, we infer that there exists $I^{*}$ sufficiently large such that for all $I \geq I^{*}$, 


$$
\begin{aligned}
{\left[L_{1}(x, y(J, x))\right.} & \left.-\vec{v}_{1}(x, y(J, x)) \cdot \nabla\right] y(J, x) \\
& +\gamma(y(J, x)) I \Psi^{*}(x)-G(y(J, x))-\sum_{j \neq 1} \sigma_{1 j}^{*}(x) y(J, x)>0
\end{aligned}
$$

for all $x \in B$ and it is 0 for all $x \in \Omega \backslash B$, where

$$
\Psi^{*}(x)=\mathcal{K}(x) \exp \left(-\int_{l_{x}(x) \cap \Omega} \gamma^{*} d l\right) .
$$

If we fix $I \geq I^{*}$, then one can find $Y(I)$ such that $\gamma\left(u_{1}\right) \Psi(0)(x) I-$ $G\left(u_{1}\right)<0$ for all $u_{1}>Y(I)$ and $Y(I)>y(J, x)$ for all $x \in \bar{\Omega}$. The constant $Y(I)$ will be the first component of our supersolution. Now, we construct the other components. For $i \neq 1$, let

$$
\sigma_{i 1}^{Y}(x)=\max _{u_{k} \in[0, Y(I)], k=2, \ldots, m} \sigma_{i 1}\left(Y(I), u_{2}, \ldots, u_{m}\right) .
$$

Let $\left(Y_{2}(I, x), \ldots, Y_{m}(I, x)\right)$ be the solution to the system of $m-1$ equations

$$
\begin{gathered}
L_{i}\left(x, u_{i}(x)\right) u_{i}(x)-\vec{v}_{i}\left(x, u_{i}(x)\right) \cdot \nabla u_{i} \\
-\sum_{j \neq i, 1} \sigma_{i j}\left(u_{i}(x)\right)\left(u_{i}(x)-u_{j}(x)\right)-\sigma_{i 1}^{Y}(x)\left(u_{i}(x)-Y(I)\right)=0, \\
u_{i}(x)=\varepsilon, \quad x \in \partial \Omega,
\end{gathered}
$$

$i=1, \ldots, m-1$, where $\varepsilon>0$ is an arbitrarily small number. Note that the systems of functions $(0, \ldots, 0)$ and $(Y(I), \ldots, Y(I))$ satisfy the conditions of Assumption 6 . Hence there exists a solution $\left(Y_{2}(x), \ldots, Y_{m}(x)\right)$ to the above system such that

$$
0 \leq Y_{i}(I, x) \leq Y(I)
$$

for all $x \in \bar{\Omega}, i \in\{2, \ldots, m\}$. From the positiveness theorem (Theorem 2.3.7, p. 65 of [17]) it follows that either $Y_{i}(I, x) \equiv \varepsilon$ or $Y_{i}(I, x)>\varepsilon$ for all $x \in \Omega$. The first possibility should be excluded as, according to the definition of $\sigma_{i 1}^{Y}(x)$, we would have

$$
-\sum_{j \neq i, 1} \sigma_{i j}(\varepsilon)\left(\varepsilon-Y_{j}(I, x)\right)-\sigma_{i 1}^{Y}(x)(\varepsilon-Y(I))>0, \quad x \in \Omega,
$$

and we would arrive at contradiction.

Now, as in the case of one equation, it is easy to prove that

$$
\left(J \psi_{B}(h, x), 0, \ldots, 0\right), \quad\left(Y(I), Y_{2}(x), \ldots, Y_{m}(x)\right)
$$

are sets of sub- and supersolutions satisfying Assumption 9.

Finally, note that for any domain $D$ lying in the light cone there exists a set $B$ such that $D \subset \subset B$ and $B$ lies in the light cone. Thus by choosing the constant $J$ appropriately we can prove the following theorem:

Theorem 6. For any $C^{2+\alpha}$ domain $D$ with $\bar{D} \subset \Omega$ such that $\bar{D}$ lies in the interior of the light cone, and any given $U>0$, there exists $I_{D U}>0$ 
such that for all $I>I_{D U}$ there exists a $C^{2+\alpha}$ solution $\left(u_{1}(x), \ldots, u_{m}(x)\right)$ to problem (24)-(26) such that $u_{1}(x)>U$ for all $x \in D$.

Appendix. Proof of Theorem 2. First we will assume the strict version of inequality (7), i.e.

$$
n\left(u_{0}\right) \cdot \mathcal{F}(u)\left(x_{0}\right)>0 .
$$

By fixing $u$ in the coefficients $a_{k j}^{i}(x, u, \partial u)$ and treating the right hand sides of (1) as given we can formally solve the system (4)-(5) to obtain the relation

$$
u=\mathcal{N}(u):=\mathcal{R}(u) \circ \mathcal{F}(u) .
$$

According to the linear theory of elliptic operators, $\mathcal{N}(u)$ is a well defined operator acting from $C^{2}(\bar{\Omega})$ to $C^{2}(\bar{\Omega})$. Moreover, it is completely continuous, as it maps into $C^{2+\alpha}(\bar{\Omega})$. Let $\mathcal{E}$ denote the Banach space of real $C^{2}(\bar{\Omega})$ functions with the natural norm

$$
\|v\|_{\mathcal{E}}:=\|v\|_{C^{2}(\bar{\Omega})}=\|v\|_{C^{0}(\bar{\Omega})}+\|v\|_{2} .
$$

According to Assumption 5 for $u$ satisfying (4)-(5)) and such that $u(x) \in \bar{\Sigma}$ for $x \in \bar{\Omega}$ we have $\|u\|_{2} \leq Q$. Define a bounded open subset of $\mathcal{E}$ by

$$
\mathcal{O}:=\left\{v \in \mathcal{E}: v: \bar{\Omega} \rightarrow \Sigma,\|v\|_{2}<Q+1\right\} .
$$

Note that by a suitable translation in the $u$-space we can assume that $\mathbf{0} \in \Sigma$. This translation does not affect the value of the constant $Q$. Thus we can make use of the Leray-Schauder Continuation Theorem (see [15], [19]).

LEMmA. Let $\mathcal{E}$ be a real Banach space and $\mathcal{O}$ a bounded open neighborhood of $\mathbf{0}$ in $\mathcal{E}$. Let $\mathcal{N}: \bar{\Omega} \rightarrow \mathcal{E}$ be a completely continuous operator such that $u \neq \lambda \mathcal{N}(u)$ for all $\lambda \in(0,1)$ and $u \in \partial \mathcal{O}$. Then the equation $u=\mathcal{N}(u)$ has a solution $u \in \overline{\mathcal{O}}$.

Due to this Lemma, Theorem 2 will be proved once we show that for all $u \in \partial \mathcal{O}$ and $\lambda \in(0,1), u \neq \lambda \mathcal{N}(u)$. Suppose to the contrary that there exist $\lambda \in(0,1)$ and $u \in \partial \mathcal{O}$ such that

$$
u=\lambda \mathcal{N}(u)=\lambda \mathcal{R}(u) \circ \mathcal{F}(u) .
$$

Then $u$ must be a solution of the problem

$$
\begin{aligned}
L_{i}(x, u(x), \partial u(x)) u_{i}(x) & =\lambda \mathcal{F}_{i}(u)(x) & & \text { for } x \in \Omega, \\
u_{i}(x) & =\lambda \phi_{i}(x) & & \text { for } x \in \partial \Omega,
\end{aligned}
$$

where $i=1, \ldots, m \geq 1$.

Note that the boundary of $\mathcal{O}$ consists of functions $v: \bar{\Omega} \rightarrow \bar{\Sigma}$ with range meeting $\partial \Sigma$ and of functions $v$ such that $\|v\|_{2}=Q+1$. However, by Assumption 3 the second group of functions is empty. Thus, if $u \in \partial \mathcal{O}$ satisfies (32), then there exists $\zeta \in \bar{\Omega}$ such that $u(\zeta) \in \partial \Sigma$. On the other hand, as 
$\Sigma$ is open and convex, $\lambda \phi(x) \in \Sigma$ for $x \in \partial \Omega$ and $\lambda \in(0,1)$ (see Assumption 4). So, $\zeta \in \Omega$. Set $r(x):=n(u(\zeta))(u(x)-u(\zeta))$, where $n(u(\zeta))$ is an outer normal at $u(\zeta) \in \partial \Sigma$. We have $r(\zeta)=0$ and $r(x) \leq 0$ for $x \in \bar{\Omega}$ (see Definition 1). As $\lambda \phi(x) \in \Sigma$ for $x \in \partial \Omega, r(x)<0$ for $x \in \partial \Omega$. Consequently, $r(x)$ attains its maximum only at inner points of $\Omega$. Hence $\partial_{j} r(\zeta)=0$, which implies that $n(u(\zeta)) \cdot\left(\partial_{j} u\right)(\zeta)=0$ for $j=1, \ldots, n$. From the assumptions of Theorem 2 it follows that $n(u(\zeta)) \cdot f(\zeta, u(\zeta), \partial u(\zeta)) \geq 0$ and there exists $h \in\{1, \ldots, m\}$ such that $n(u(\zeta)) \cdot u(\zeta)=u_{h}(\zeta)$. Thus we have

$$
\begin{aligned}
L_{h}(\zeta, u(\zeta)) r(\zeta) & =L_{h}(\zeta, u(\zeta)) u_{h}(\zeta)=\lambda \mathcal{F}_{h}(\zeta, u(\zeta), \partial u(\zeta)) \\
& =\lambda n \cdot \mathcal{F}(\zeta, u(\zeta), \partial u(\zeta))>0 .
\end{aligned}
$$

It follows that there exists a ball $B$ with center at $\zeta$ and $\bar{B} \subseteq \bar{\Omega}$ such that

$$
L_{h}(x, u(x)) u_{h}(x)=\lambda \mathcal{F}_{h}(x, u(x), \partial u(x)) \geq 0, \quad x \in \bar{B} .
$$

From the maximum principle it follows that $u_{h}(x) \equiv 0$ for $x \in \bar{B}$. Consequently, $L(\zeta, u(\zeta)) u_{h}(\zeta)=0$, which contradicts inequality (33). According to the last Lemma we obtain a solution of (4)-(5) belonging to $C^{2+\alpha}(\bar{\Omega})$.

Now, suppose that $n\left(u_{0}\right) \cdot \mathcal{F}(u)\left(x_{0}\right)=0$ at some $u_{0} \in \partial \Sigma$. Replace $\mathcal{F}_{i}$ by $\mathcal{F}_{i \varepsilon}=\mathcal{F}_{i}+\varepsilon u_{i}, \varepsilon>0$, on the right hand side of (4). Then, if $\mathbf{0} \in \Sigma$, the assumptions of Theorem 1 will imply that $n\left(u_{0}\right) \cdot \mathcal{F}_{\varepsilon}(u)\left(x_{0}\right)>0$ for all $u_{0} \in \partial \Sigma$ such that $u\left(x_{0}\right)=u_{0}$. Thus, for $\varepsilon_{\kappa}:=1 / \kappa, \kappa \in \mathbb{N}$, we obtain a sequence of $C^{2+\alpha}(\bar{\Omega})$ solutions to the problems

$$
\begin{aligned}
L_{i}\left(x, u_{\varepsilon}(x), \partial u_{\varepsilon}(x)\right) u_{i \varepsilon}(x) & =\mathcal{F}_{i \varepsilon}\left(u_{\varepsilon}\right)(x) & & \text { for } x \in \Omega, \\
u_{i \varepsilon}(x) & =\phi_{i}(x) & & \text { for } x \in \partial \Omega .
\end{aligned}
$$

Due to the compact embedding of $C^{2+\alpha}$ in $C^{2+\tau}$ for any $\tau \in(0, \alpha)$ we can choose a subsequence converging to some function $u$ in $C^{2+\tau}(\bar{\Omega})$ which is a solution of (4)-(5). But such a solution is also of class $C^{2+\alpha}$. The proof of Theorem 2 is now complete.

\section{References}

[1] H. Amann, Invariant sets and existence theorems for semilinear parabolic and elliptic systems, J. Math. Anal. Appl. 65 (1978), 432-467.

[2] I. Bakelman, Convex Analysis and Nonlinear Geometric Elliptic Equations, Springer, Berlin, 1994.

[3] J. Bebernes, K. Chueh and W. Fulks, Some applications of invariance for parabolic systems, Indiana Univ. Math. J. 28 (1979), 269-277.

[4] K. Chueh, C. C. Conley and J. Smoller, Positively invariant regions for systems of nonlinear diffusion equations, ibid. 26 (1977), 373-392.

[5] W. Eckhaus, A. van Harten and Z. Peradzyński, A singularly perturbed free boundary problem describing a laser sustained plasma, SIAM J. Appl. Math. 45 (1985), 1-31. 
[6] P. Fife and M. Tang, Comparison principles for reaction-diffusion systems: irregular comparison functions and applications to questions of stability and speed of propagation of disturbances, J. Differential Equations 40 (1981), 168-185.

[7] D. Gilbarg and N. Trudinger, Elliptic Partial Differential Equations of Second Order, Springer, 1983.

[8] A. Guetter, A free boundary value problem in plasma containment, SIAM J. Appl. Math. 49 (1989), 99-115.

[9] J. Hernández, Some existence and stability results for solutions of reaction-diffusion systems with nonlinear boundary conditions, in: Nonlinear Differential Equations, P. de Mottoni and L. Salvadori (eds.), Academic Press, New York, 1981, 161-173.

[10] B. Kaźmierczak, Existence theorems of elliptic partial differential equations of reaction-diffusion type, Arch. Mech. 47 (1995), 117-124.

[11] B. Kaźmierczak and Z. Peradzyński, Heteroclinic solutions for a system of strongly coupled ODEs, Math. Methods Appl. Sci. 19 (1996), 451-461.

[12] A. I. Košelev and C. I. Chelkak, Regularity of Solutions to Quasilinear Elliptic Equations, Teubner, Leipzig, 1985.

[13] O. A. Ladyzhenskaya and N. N. Ural'tseva, Linear and Quasilinear Elliptic Equations, Academic Press, New York, 1968.

[14] J. Nečas, Introduction to the Theory of Nonlinear Elliptic Equations, Teubner-Texte zur Math. 52, Teubner, Leipzig, 1983.

[15] L. Nirenberg, Topics in Nonlinear Functional Analysis, Courant Inst. Lect. Notes, New York Univ., New York, 1974.

[16] M. Plum, Shape-invariant bounds for reaction-diffusion systems with unequal diffusion coefficients, J. Differential Equations 73 (1988), 82-103.

[17] M. Protter and H. Weinberger, Maximum Principles in Differential Equations, Springer, New York, 1984.

[18] D. Sattinger, Topics in Stability and Bifurcation Theory, Lecture Notes in Math. 309, Springer, 1973.

[19] K. Schmitt, Boundary value problems for quasilinear second order elliptic equations, Nonlinear Anal. 2 (1978), 263-309.

[20] J. Smoller, Shock Waves and Reaction-Diffusion Equations, Springer, 1983.

[21] M. Valencia, On invariant regions and asymptotic bounds for semilinear partial differential equations, Nonlinear Anal. 14 (1990), 217-230.

Institute of Fundamental Technological Research

Świętokrzyska 21

00-049 Warszawa, Poland

E-mail: bkazmier@ippt.gov.pl

Received on 14.3.2001;

revised version on 1.10.2001 